\title{
Problem of a quantum particle in a random potential on a line revisited ${ }^{*}$
}

\author{
O. K. Vorov ${ }^{\dagger}$ and A. V. Vagov ${ }^{\ddagger}$
}

(20 September 1994)

\begin{abstract}
The density of states for a particle moving in a random potential with a Gaussian correlator is calculated exactly using the functional integral technique. It is achieved by expressing the functional degrees of freedom in terms of the spectral variables and the parameters of isospectral transformations of the potential. These transformations are given explicitly by the flows of the Korteweg-de Vries hierarchy which deform the potential leaving all its spectral properties invariant. Making use of conservation laws reduces the initial Feynman integral to a combination of quadratures which can be readily calculated. Different formulations of the problem are analyzed.

PACS: 71.20.-b,71.20.Ad,71.25.Mg,71.55.Jv
\end{abstract}

Typeset using REVTEX

*Preprint ANU-RsphysSE-20994

Received 20 September 1994

†Theoretical Department, School of Physics, University of New South Wales, Sydney, NSW 2052, Australia

$\ddagger$ Theoretical Physics Department, Research School of Physical Sciences and Engineering, The Australian National University, Canberra, ACT 0200, Australia 
The problem of a quantum particle moving in a random potential has been of interest for a long time. It has numerous applications to solid state physics [1], particularly to the electron structure of heavily doped semiconductors. Various modifications of the problem of the calculation of the Green's function and Density of States (DOS) averaged over the ensemble of random potentials arise (Thouless and Edwards [2]) in connection with the Anderson localization [3], in the physics of amorphous materials, particularly glasses [4], in considerations of the conductivity in wires and mesoscopic objects [5], in the phenomena of broadening of resonance lines in solids [6,7], in a number of problems connected to the Quantum Hall effect [8] and many other problems related to disorder [9 11.

In particular, the one-dimensional problem with a random potential having a white-noise correlator has been considered in a large number of works. The case when the potential is arbitrary but varies very slowly compared with the wave function of an electron was considered by Kane [12]. To calculate the DOS for the reverse situation with randomly distributed $\delta$-functional wells, Frish and Lloyd employed stochastic equations [13]. In the subsequent studies of the more general case of an arbitrary uncorrelated potential the methods of "minima counting" (Halperin and Lax [14]) and "zeros counting" were used (Lifshits [6], Halperin [15]). Zittars and Langer formulated it in the Feynmann path integral language [16]. That approach was further developed by Parisi who used the replica trick [4] and alternatively by Efetov by means of auxiliary Grassmannian fields [17]. Berezinski rigorously considered the problem by a diagram summation technique [18]. Luttinger and Waxler considered special potential configurations ( $\delta$-functional scatterers) by the functional integral technique [19].

However, even for the 1D problem with a white-noise correlator the average density of states $\eta(E)$ has not been found exactly due to technical difficulties specific for the methods used. Most of the results obtained so far give only the "tail" behavior of the density of states, i.e., the DOS asymptotics at large negative energies $E$ which is found to be $\sim \exp \left(-|E|^{3 / 2}\right)$.

A nonperturbative approach to the problem presented here uses essentially the ideas of Inverse Scattering Method (ISCM) for solving nonlinear evolution equations proposed in the celebrated works by Gardner, Greene, Kruskal and Miura [20], and Lax [21] and developed in Refs. 22 27]. Methodically, what is done in this work is a kind of inversion of this 'diamond of mathematical physics'. Here, the Schrödinger equation is to be solved and we use the hierarchy of Korteweg-de Vries $(\mathrm{KdV})$ equations to find a parametrization of the random 
potential.

DOS as a function of energy $E$ averaged over the ensemble of random potentials with a Gaussian white noise correlator can be found from the functional integral (FI):

$$
\begin{gathered}
\langle\eta(E)\rangle=\frac{1}{\pi} \lim _{\delta \rightarrow 0} \operatorname{Im}\left[\operatorname{Tr}\left\langle G_{E-i \delta}\right\rangle\right]= \\
\frac{1}{\pi Z} \lim _{\delta \rightarrow 0} \int D[U(x)] e^{-a^{3} \int U(x)^{2} d x} \operatorname{Im}\left[\operatorname{Tr} G_{E-i \delta}\{U(x)\}\right],
\end{gathered}
$$

where $G_{E}\{U(x)\}$ is the Green's function of a particle in the potential $U(x), D[U(x)]$ is a functional integration measure, $\langle\ldots\rangle$ means an ensemble average. The partition function $Z=$ $\int D[U(x)] e^{-a^{3} \int U(x)^{2} d x}$ is the normalization constant, the trace means the integral $\operatorname{Tr}[G]=$ $\int_{-\infty}^{\infty} d x G\left(x, x^{\prime}=x\right)$ and the constant $a$ is the ensemble correlation length of the dimensionality $[x]=\left[E^{-1 / 2}\right]$. We use the units in which $\hbar^{2} / m=1$. Due to the Gaussian weight only quadratically normalizable configurations enter the path integral (四):

$$
\int_{-\infty}^{+\infty} U^{2}(x) d x<\infty
$$

Since the functions $U(x)$ model the real impurity potentials, which are smooth, it is assumed that $U(x)$ can be differentiated an appropriate amount of times.

The Green's function $G$ in (11) corresponds to the eigenvalue problem for the Schrödinger operator $\hat{L}$

$$
\begin{gathered}
{[E-\hat{L}] G_{E}\left(x, x^{\prime}\right)=\delta\left(x-x^{\prime}\right), \quad \hat{L} \psi_{k}(x)=\varepsilon_{k} \psi_{k}(x),} \\
\hat{L}=-\frac{1}{2} \frac{d^{2}}{d x^{2}}+U(x)
\end{gathered}
$$

with the boundary conditions specified below. To average the DOS one has to solve these equations for every particular potential configuration $U(x)$ entering the path integral and that appears to be the main difficulty in a straightforward approach. Let us note that the present problem (11) is similar to the one considered in the Random Matrix Theory (RMT) 28].

The boundary conditions for Eq.(3) are dictated by requirement (2) and are typical for the scattering problem 1 . The particle is moving on a line in a square normalizable potential vanishing at infinity. Motion at $E>0$ is unbounded, and the spectrum remains continuous

\footnotetext{
${ }^{1}$ Actually, the stricter condition $\int|U(x)|(1+|x|) d x<\infty$ is required for rigorous validity of the following consideration. It means the potential vanishes rapidly at infinity.
} 
as for a free particle. Therefore the density of states at $E>0$ remains unaffected by such a potential and equals const $\times E^{-1 / 2}$. Thus, we are interested in DOS for negative energies where all states are bounded and have discrete eigenvalues. So the boundary conditions for Eq.(3) are assumed to be $\psi( \pm \infty)=0$.

Our method employs an accurate change of variables in the path integral (11). The idea is to use the spectral data of a potential as a set of new variables instead of the potential itself. The spectral data alone cannot describe a potential configuration uniquely, because a lot of other configurations entering the integrand (1) yield the same spectral properties. To account for all the potentials from the same isospectral family one needs to have an additional set of variables which describe all the spectrally equivalent configurations. The generators of the group of the isospectral transformations of the Schrödinger operator $\hat{L}$ are the Lax operators $\hat{A}_{2 j+1}$ obeying the commutation relations [21]

$$
\left[d / d \tau_{2 j+1}+\hat{A}_{2 j+1}, \hat{L}\right]=0, \quad\left[\hat{A}_{2 j+1}, \hat{A}_{2 j^{\prime}+1}\right]=0
$$

where $j$ is a positive integer and the "times" $\tau_{2 j+1}$ play the role of parameters and count the members of an isospectral family of potentials. The relations (雨) enforce the set nonlinear equations for $U(x)$ (KdV hierarchy)

$$
U_{\tau_{1}}=U_{x}, \quad U_{\tau_{3}}=-U_{x x x}+12 U U_{x}, \quad \ldots
$$

(the notation $U_{a} \equiv \partial U / \partial a$ is used). The role of parameters $\tau_{1}, \tau_{3}, \ldots$ is analogous to that of the angles of unitary transformations in the language of the RMT [28].

The essence of the ISCM theory (see, e.g., [22]- [24]) which is important in the present context can be reformulated in the following rigorous statements:

(i) Each square normalizable potential configuration, provided that condition (2) is fulfilled (see footnote 1) Ecan be characterized by an integer number of bound states $N \geq 0$, with the set of energies $\varepsilon_{i}=\kappa_{i}^{2}, 1 \leq i \leq N$,

(ii) Each solution of the KdV hierarchy consists of a soliton component $U_{s}$ controlled by the finite number of discrete eigenvalues of the problem (3) and a radiation component $U_{r}$

2 This requirement is important for the establishment of analytical properties of solutions of $(2)$ that used in the ISCM [24]. 
described by a continuous set of variables related to the reflection coefficient $R$. The soliton and radiation degrees of freedom are independent.

(iii) The possibility for a configuration to evolve according to the $\mathrm{KdV}$ flows (5) gives rise to a corresponding volume of isospectral transformations associated with it. The total weight of the configurations with definite set $\left\{\varepsilon_{i}, N\right\}$ in the ensemble is proportional to this volume.

Each of the potential configurations that enter our functional integral viewed as a solution of the KdV hierarchy (5) and it can be obtained from the trivial configuration $U(x) \equiv 0$ by means of the Bäcklund transformations or by Hirota's direct method [25].

In view of the above classification (i)-(iii), the two following formulations of the problem of calculation of the average DOS are natural:

A. The total number of bound states allowed for a given ensemble is fixed $N\{U(x)\}=$ $N=$ const for all particular realizations of the random potential. In that case, one should calculate the total level density $\eta(E)$.

B. The total number of discrete levels is not fixed by physical conditions, and calculation of the "reduced" DOS, i.e., the density per a length unit, $\eta^{\prime}(E)=d \eta(E) / d x$, is reasonable. In this case, one should keep the total "concentration" of bound states on the line $c$ fixed.

We shall consider both cases, implying that a particular choice should follow from the physical requirements for a given system under consideration.

We evaluate the functional integral (1) using the exact parametrization of the potential viewed as a solution of the $\mathrm{KdV}$ hierarchy (5), that is given by the complete set of new variables [23, 26, 24]

$$
U(x)=U(x, \underbrace{\varepsilon_{i},\{\mathcal{P}(k)\}}_{\text {actions }}, \underbrace{\theta_{i},\{\mathcal{Q}(k)\}}_{\text {angles }})
$$

where $\varepsilon_{j}<0$ are the energies of the "solitons" (bound states), $\theta_{j}$ are soliton phases related to the soliton positions $X_{j}$ as $\theta_{j}=\left|2 \varepsilon_{j}\right|^{1 / 2} X_{j}=\sum_{m=0}^{\infty}\left|2 \varepsilon_{j}\right|^{m+1 / 2} \tau_{2 m+1}$, and the continuous set $\mathcal{Q}(k)$ defined at positive energies $\varepsilon=2 k^{2}$ describes the isospectral transformations of the radiation part. The radiational actions set $\mathcal{P}(k)$ is related to the reflection coefficient: $\mathcal{P}(k)=-\frac{2 k}{\pi} \log \left(1-|R(k)|^{2}\right)$. As the KdV hierarchy is a fully integrable Hamiltonian system [23], Eq.(6) is the canonical transformation from the potential variables $U(x)$ with given number $N$ of bound states to the action-angle type variables [26]. The canonical Hamiltonian structure of the KdV hierarchy is governed by the standard Poisson brackets for the new variables 
$\left\{\varepsilon_{j}, \theta_{j^{\prime}}\right\}=\delta_{j j^{\prime}}, \quad\left\{\mathcal{P}(k), \mathcal{Q}\left(k^{\prime}\right)\right\}=\delta\left(k-k^{\prime}\right)$ with all other brackets zero [27]. The Jacobean of the canonical transformation $U(x) \rightarrow\left\{\varepsilon_{j}, \theta_{j}, \mathcal{P}(k), \mathcal{Q}_{j}\right\}$ is unity [29] and the measure is just a product of differentials of the new variables. This transformation is correct because only smooth configurations are to be counted in the Feynmann integral and the problem with discontinuous paths typical in the field theories is irrelevant in the present context [30]. Thus we can express the functional measure in (1) in terms the new variables as follows

$$
\begin{gathered}
\int D[U(x)] \Phi\{U(x)\}=\int \prod_{x} d U(x) \Phi\{U(x)\}= \\
\int \prod_{j=1}^{\infty} d \varepsilon_{j} d \theta_{j} D[\mathcal{P}(k)] D[\mathcal{Q}(k)] \Phi\left\{U\left(x, \varepsilon_{j}, \theta_{j},\{\mathcal{P}, \mathcal{Q}\}\right)\right\},
\end{gathered}
$$

where $\Phi$ is the integrand of Eq.(11). Let us stress that while we integrate over parameters of the evolution of the $\mathrm{KdV}$ equations we count all but the static potential configurations, and no real time evolution is involved in the problem at all.

The next advantage of the soliton language is the possibility to rewrite the Gaussian weight in Eq. (11) compactly using the second conservation law for the KdV hierarchy 24]

$$
\int_{-\infty}^{\infty} U(x)^{2} d x=\sum_{i}^{N} \frac{4}{3}\left|2 \varepsilon_{i}\right|^{\frac{3}{2}}+\int_{0}^{\infty} \frac{2 k^{2}}{\pi} \log \left(1-|R(k)|^{2}\right) d k .
$$

First we consider the case $\mathbf{A}$ when the total number $N$ of bound states is fixed. The expression (11) with the account for (7),(8) read as follows

$$
\begin{gathered}
\langle\eta(E)\rangle=\frac{I_{N}}{Z_{N}}=\frac{1}{Z_{N} N !} \int D[\mathcal{P}(k)] D[\mathcal{Q}(k)] \int \prod_{i} d \varepsilon_{i} d \theta_{i} \\
\left.\times \exp \left[-4 a^{3} \sum_{i} \frac{\varepsilon_{i}^{\frac{3}{2}}}{3}-a^{3} \int_{0}^{\infty} k d k \mathcal{P}(k)\right)\right] \sum_{i=1}^{N} \delta\left(E-\varepsilon_{i}\right),
\end{gathered}
$$

with

$$
\begin{aligned}
Z_{N} & =\frac{1}{N !} \int D[\mathcal{P}(k)] D[\mathcal{Q}(k)] \int \prod_{i} d \varepsilon_{i} d \theta_{i} \\
& \times \exp \left[-\frac{4}{3} a^{3} \sum_{i}\left|2 \varepsilon_{i}\right|^{3 / 2}-a^{3} \int_{0}^{\infty} k \mathcal{P}(k) d k\right] .
\end{aligned}
$$

The $(N !)^{-1}$ factor appears due to symmetry reasons. The integration over the "radiation" variables $\{\mathcal{P}(k), \mathcal{Q}(k)\}$ corresponds to the continuous spectrum of ISCM and is still continual. It is important that when expressed through the conserving quantities of the KdV equations, the integrands in (9) depend only on the action variables $\left\{\varepsilon_{i}, \mathcal{P}(k)\right\}$.

The functional integration over $\mathcal{P}(k)$ and $\mathcal{Q}(k)$ factorizes giving the same functional constant $\int D[\mathcal{P}(k)] D[\mathcal{Q}(k)] \exp \left[-a^{3} \int_{0}^{\infty} k \mathcal{P}(k) d k\right]$ for both the numerator $I_{N}$ and the denominator 
$Z_{N}$ in the Eq.(9). It is the manifestation of the fact discussed above that the radiation component of the potential $U_{r}(x)$ does not yield bound states and does not affect the "soliton energies" $\varepsilon_{i}$. Hence it does not influence the DOS for $E<0$. To define the divergent integrations over the soliton phases $\theta_{i}$ we introduce the length of the system to play the role of a cutting parameter. In order to do so we rewrite the remaining integrals in the Eq.(9) in terms of the soliton coordinates $X_{i}=\theta_{i} /\left|2 \varepsilon_{i}\right|^{1 / 2}$ [24]. The Jacobean for this transformation is $\prod_{i=1}^{N} \sqrt{2\left|\varepsilon_{i}\right|}$ and we obtain from (9)

$$
\langle\eta(E)\rangle=\frac{\prod_{i}^{N}\left[\int_{0}^{\infty} d \varepsilon_{i}\left|2 \varepsilon_{i}\right|^{\frac{1}{2}} \int_{-\infty}^{\infty} d X_{i}\right] \exp \left[-\sum_{i=1}^{N} \frac{4\left|2 \varepsilon_{i}\right|^{3 / 2} a^{3}}{3}\right] \sum_{i=1}^{N} \delta\left(E-\varepsilon_{i}\right)}{\prod_{i}^{N}\left[\int_{0}^{\infty} d \varepsilon_{i}\left|2 \varepsilon_{i}\right|^{\frac{1}{2}} \int_{-\infty}^{\infty} d X_{i}\right] \exp \left[-\frac{4}{3} a^{3} \sum_{i=1}^{N}\left|2 \varepsilon_{i}\right|^{3 / 2}\right]}
$$

It is interesting that similar expressions are normally obtained in the Gaussian ensemble of random matrices (except for the change $\varepsilon_{i}^{2} \rightarrow \varepsilon_{i}^{3 / 2}$ ) [28]. However, the level repulsion is absent here contrary to the RMT, where it usually appears in the form of the factor $\prod_{i \neq j}\left|\varepsilon_{i}-\varepsilon_{j}\right|$ [28]. This property is specific for the the infinite length line, where one can have a potential configuration with two bound states with an arbitrary small difference between corresponding eigenvalues. Now all the integrals over the isospectral variables $X_{i}$ cancel, and Eq. (10) is reduced to the combination of quadratures which gives the final result for the local DOS

$$
\text { Case } \mathbf{A}:\langle\eta(E)\rangle=4 a^{3} N \sqrt{|2 E|} e^{-\frac{4}{3} a^{3}|2 E|^{3 / 2}} .
$$

We turn now to the case $\mathbf{B}$, where the number of bound states is not fixed from the very beginning. Due to the above classification every potential configuration belongs to one of the classes which differ by the number of the solitons $N$. And instead of (9) we can write the following expression for the averaged DOS

$$
\langle\eta(E)\rangle=\frac{D_{0} I_{0}+D_{1} I_{1}+\ldots+D_{N} I_{N}+\ldots}{D_{0} Z_{0}+D_{1} Z_{1}+\ldots+D_{N} Z_{N}+\ldots}
$$

with the integrals $I_{N}, Z_{N}$ given by Eq.(9) 3. The dimensional functional constants $D_{N}$ correspond to the relative probabilities for the N-soliton configurations to occur. To evaluate the expression (12) we recall that creation of a new $N+1$ 'th soliton in addition to $N$ existing ones gives rise to an additional degree of freedom. It is related to "translation" of this extra

\footnotetext{
${ }^{3}$ Note the formal similarity between present classification and those arising in the nonperturbative field theories in discussions of the multi-instanton configurations [31].
} 
potential well along the $X$ axis. It enlarges the weight of $N+1$-soliton potential configurations by a formally divergent factor $\int d X$. Thus the configurations containing the number of solitons less than a given integer $N$ have a zero measure in the ensemble compared to $N$-soliton configurations, and Eq.(12) reduces to

$$
\langle\eta(E)\rangle=\lim _{N \rightarrow \infty} \frac{D_{N} I_{N}}{D_{N} Z_{N}}=\lim _{N \rightarrow \infty} \frac{I_{N}}{Z_{N}} .
$$

The fact that only the terms with maximum $N$ survive in Eq.(12) can be proved explicitly by considering a discretized version of FI. The probabilities for $N$-soliton potentials to appear are evaluated with the aid of the expression for the number of bound states (solitons) $N=$ $\frac{1}{\pi} \int_{U<0} \sqrt{|U(x)|} d x[32]$ valid for large $N$ which we need in Eqs.(12,,13).

The total average DOS is related to the reduced DOS (i.e., per unit length) $\eta^{\prime}(E)$ by the obvious relation $\eta(E)=\int d x \eta^{\prime}(E)$. The reduced DOS is independent of $x$ due to translational invariance. After the simple transformations we obtain the result

$$
\text { Case } \quad \mathbf{B}: \quad\left\langle\eta^{\prime}(E)\right\rangle=4 a^{3} c \sqrt{2|E|} e^{-a^{3} \frac{4}{3}|2 E|^{3 / 2}}
$$

The total concentration of the bound states on the line, $c$, mentioned above, appears here as a natural limit $N / \int d x \rightarrow c$ at very large $N$.

Note that both the expressions (11) and (14) for the DOS correspond to the "local" density which is connected to the so called "cumulative" density of states $\mathcal{N}(E)$ by the relation $\eta(E)=d \mathcal{N}(E) / d E$ [14,16]. The value $\mathcal{N}(E)$ is the number of levels with energy less than a given value $E$. Expressions (11) and (14) are similar in form to those obtained elsewhere [13,14 for the asymptotic of the DOS in the large $|E|$ limit (band tails). However in the present approach no approximation has been used, and therefore the result is valid for all energies.

To summarize, in this Letter we presented a nonperturbative method to calculate the DOS in a random potential. Using the exact classifications of the potential configurations in the functional integral in terms of the inverse scattering method, we obtain an analytical result for the DOS random potential with a white-noise correlator. No restriction by particular energy regions are needed within this approach.

This method can be applied, though with some modifications, to a calculation of the averaged Green's function $\left\langle G\left(x, x^{\prime}\right)\right\rangle$ (two-point correlator). This will be presented in a forthcoming paper. 
Concluding, we note that the present approach is based on a deep connection between the 1D Schrödinger equation and the exactly solvable nonlinear KdV hierarchy and hence can be directly applied to 1D problems only. But the method of using isospectral transformations to fulfill the averaging over the field ensemble looks quite fruitful and we believe that it can be done in a number of related problems in more than one dimensions in particular for the calculation of the DOS in a 2D random potential in the presence of magnetic field. It can also be used in random matrix theory. Work in these directions is now in progress.

Comment added after submission: After our paper has been submitted to this database we were made known that a similar approach to the problem was discussed earlier by B.N.Shalaev in Fiz. Tverd. Tela (Leningrad) 323586 (December 1990), [Sov. Phys. Solid State 32 (12) 2079 (1990)] (Ref. [33). However, important aspects of considerations and the results of the two works are different. We are grateful to R.H. McKenzie for making us known about the work by B.N.Shalaev. 


\section{REFERENCES}

[1] V.L.Bonch-Bruevich, The Electronic Theory of Heavily Doped Semiconductors, N.Y., American Elsevier, 1966; J.M.Ziman, Models of Disorder, Cmbr.Uni., Cambridge, 1990; P.V.Mieghem, Rev. Mod. Phys. 64, 755 (1992).

[2] D.J.Thouless, Phys.Rev.Lett. 39, 1167 (1977); J. Phys. C5, 77 (1972), ibid. C6, (1973); J.T.Edwards and D.T.Thouless, J.Phys. C5, 807 (1972).

[3] P.W.Anderson, Phys.Rev. 109, 1492 (1958).

[4] G.Parisi, Field Theory, Disorder and Simulations, Wrld. Sci., Singapore, 1992, and references therein.

[5] S.Iida, H.A.Weidenmüller and J.A.Zuk, Phys.Rev.Lett. 64, 583 (1990) and Ann.Phys. (NY) 200, 219 (1990).

[6] I.M.Lifshitz, Sov. Physics Uspekhi, 7, 549 (1965), [Sov.Usp.Fiz.Nauk 83, 617 (1964)].

[7] A.M.Stoneham, Rev.Mod.Phys. 41, 82 (1969).

[8] V.Sa-yakanit and H.R.Glyde, in Proc. of the Third International Conference on Path integral from meV to $\mathrm{MeV}$, Wrld.Sci., Singapore, 1989, p. 163; K.Esfarajani, H.R.Glyde, and V.Sa-yakanit, ibid. p.176, see also R.E.Prange, in Quantum Hall Effect, R.E.Prange and S.M.Girvin eds, Springer-Verlag, 1987.

[9] F.J.Wegner, Phys.Rev. B19, 783 (1979); K.Ziegler, Phys.Lett. 92A, 339 (1982).

[10] Ya.Gol'dshtein, S.A.Molchanov and L.A.Pastur, Func.Anal. \& Appl.11, N1, 1 (1977).

[11] B.D.Simons and B.L.Altshuler, Phys.Rev.Lett. 70, 4063 (1993); A.G.Aronov and P.Wölfe, ibid. 72, 2239 (1994).

[12] E.O.Kane, Phys.Rev. 131, 79 (1963).

[13] H.L.Frisch and S.P.Lloyd, Phys.Rev. 120, 1175 (1960); B.Halperin, Phys.Rev. 139, A104 (1965); T.P.Eggarter, Phys.Rev. B5, 3863 (1972).

[14] B.I.Halperin and M.Lax, Phys.Rev. 148, 722 (1966).

[15] B.I.Halperin, Adv.Chem. Phys. 13, 123 (1967). 
[16] J.Zittars and. J.S.Langer, Phys.Rev. 148, 741 (1966).

[17] K.B.Efetov, Adv.Phys. 32, 53 (1983).

[18] V.L.Berezinskii, Zh.Eksp.Teor.Fyz. 65, 1251 (1973) [Sov.Phys.JETP 38, 620 (1974)]

[19] J.M.Luttinger and R.Waxler, Ann.Phys. 175, 319 (1986).

[20] C.S.Gardner, J.M.Greene, M.D.Kruskal and K.K.Miura, Phys. Rev. Lett. 19, 1095 (1967).

[21] P.D.Lax, Comm. Pure Appl. Math. 21, 467 (1968).

[22] V.E.Zakharov and A.B. Shabat, Func.Anal. \& Appl. 8, 43 (1974).

[23] V.E.Zakharov and L.D.Faddeev, Func.Anal. \& Appl. 5, 18 (1971).

[24] A.C.Newell, Solitons in Mathematics and Physics, Philadelphia, SIAM, 1985.

[25] H.D.Wahlquist and F.B.Estabrook, Phys.Rev.Lett. 31, 1386 (1975); R.Hirota, Phys.Rev. lett. 27, 1192 (1972).

[26] H.Flaschka and A.C.Newell, in Dynamical systems, Theory and Applications, J.Moser ed., Lecture Notes in Physics 38, Schpringer-Verlag, N.Y., 1975, p.355.

[27] L.D.Faddeev and L.A.Takhtajan, Lett. Math. Phys. 10, 183 (1985).

[28] Statistical Theories of Spectra: Fluctuations. C.E.Porter ed., Acad. Press, N.Y. \& London, 1965; M.L.Mehta, Random Matrices, 2nd ed., Boston, Acad. Press, 1991, see also M.R.Zirnbauer, J.J.M.Verbaarschot, and H.A.Weidenmüller, Nucl.Phys. A411, 161 (1983).

[29] H.Goldstein, Classical Mechanics, Cambridge, Mass., Addison-Wesley, 1953.

[30] R.Bullough, D.J.Pilling and J.T.Timonen, in Dynamical Problems in Soliton Systems, S.Taneko ed., Springer, Berlin 1985, p.105; K.Sasaki, ibid. p.122.

[31] A.M.Polyakov, Nucl.Phys.B 120, 429 (1977).

[32] V.I.Karpman and V.P.Sokolov, Zh.Eksp.Teor.Fiz. 54, 1568 (1968) [Sov.Phys.JETP 27, 839 (1968)].

[33] B.N.Shalaev, Fiz. Tverd. Tela (Leningrad) 323586 (December 1990), [Sov. Phys. Solid 
State 32 (12) 2079 (1990)]. 